\title{
Dictynna
}

Dictynna

Revue de poétique latine

13 | 2016

Varia

\section{Die Philologie von unten. Das athematische Lesen und der retour sur soi-même}

\section{Jürgen Paul Schwindt}

\section{OpenEdition}

1 Journals

\section{Édition électronique}

URL : http://journals.openedition.org/dictynna/1311

DOI : 10.4000/dictynna.1311

ISSN : 1765-3142

\section{Référence électronique}

Jürgen Paul Schwindt, « Die Philologie von unten. Das athematische Lesen und der retour sur soimême », Dictynna [En ligne], 13 | 2016, mis en ligne le 20 décembre 2016, consulté le 10 septembre 2020. URL : http://journals.openedition.org/dictynna/1311 ; DOI : https://doi.org/10.4000/dictynna 1311

Ce document a été généré automatiquement le 10 septembre 2020.

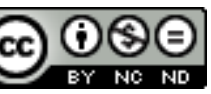

Les contenus des la revue Dictynna sont mis à disposition selon les termes de la Licence Creative Commons Attribution - Pas d'Utilisation Commerciale - Pas de Modification 4.0 International. 


\title{
Die Philologie von unten. Das athematische Lesen und der retour sur soi-même
}

\author{
Jürgen Paul Schwindt
}

\author{
Für Alain Deremetz, \\ den Liebhaber der \\ katabatischen Muse(n) ${ }^{1}$
}

Man kann in diesen Tagen und Wochen in Deutschland eine Erfahrung machen, die für Vertreter einer mittleren Generation, also diejenigen unter uns, die zu jung sind, um 68 als einschneidendes Erlebnis erfahren zu haben, und doch alt genug, um Vergleiche anstellen zu können, einigermaßen neu und ungewöhnlich ist. Genau genommen, ist es eine doppelte Erfahrung, die man machen kann, wenn man an einer deutschen Universität im Herbst 2015 über etwas spricht, über das wir, glaube ich, sprechen müssen, wenn wir uns über „Prinzipien und Methoden“ der klassischen und modernen Literaturwissenschaften verständigen wollen: Ich meine die Philologie. Da sitzt eine kleine Gruppe von Germanisten, Anglisten, Romanisten, Slavisten und Klassischen Philologen zusammen und will etwas gemeinsam machen. Jemand schlägt vor, man solle etwas zum Traditionsgedanken und, da der Traditionsgedanke, wie das Wort ja schon sagt, etwas sehr Europäisches sei, zu Europa machen. Schnell kommt der Einwand, Traditionen gebe es schließlich auch und gerade außerhalb Europas; auch sei es einigermaßen kühn, in einer Phase, da Europa sich unter dem Einfluß gewaltiger Migrationsbewegungen zu wandeln beginne, alteuropäische Traditionsbestände zum Thema eines gemeinsamen Forschungsunternehmens zu machen. Das sei doch etwas konservativ. Die Rückfrage, wer im Raum Hindi, Mandarin, Urdu oder das klassische Südarabisch spreche, verhallt ungehört. Nun gut, besinnen wir uns auf den kleinsten gemeinsamen methodischen Nenner, die Philologie. Aber nein, ist das nicht auch viel zu eng gedacht? Ist „philology“ im angloamerikanischen Raum nicht geradezu peiorativ besetzt? Und dient das Label der "Philologie“ nicht auch gerade in Deutschland vielen dazu, ihre Ideen von Weltflucht und kontemplativer Gegenstandsbehandlung zu pflegen? 
2 Im September 2015 ist in der Frankfurter Allgemeinen Zeitung genau hierüber ein Streit entbrannt. Und dieser Streit hat auch etwas mit Lille zu tun. Christoph König ist ein bekannter deutscher Germanist mittleren Alters. Er hat in einem Beitrag für die geisteswissenschaftliche Beilage der FAZ daran erinnert, daß Philologie im Kern so etwas ist wie die Begegnung mit der Reflexivität eines Textes, die - vom Philologen verstanden sein will ${ }^{2}$. Königs Kronzeuge ist ein Mann, der in den letzten fünfzig Jahren wie kein zweiter für die deutsch-französische Allianz in der Pflege und Ausübung einer „Kritischen Philologie“ gestanden hat, der ,homme Lillois‘ Jean Bollack.

3 Zwei Wochen später meldete sich eine scharfsinnige, etwas jüngere Frau, Julika Griem, Professorin für Anglistik an der Johann Wolfgang Goethe-Universität in Frankfurt, zu Wort $^{3}$. Ihr Text ist nicht nur eine detaillierte Kritik an Gestus und Begrifflichkeit des germanistischen Kollegen, sondern auch eine Kampfansage an alle, die Philologie außerhalb des institutionellen, inter- und transdisziplinären Referenzrahmens zu denken versuchen. In Königs Rede will sie das Programm einer atavistischen Auratisierung der Geltungsansprüche von Philologie erkennen. Diese könne immer nur in sozialen Zusammenhängen eine sinnvolle Praxis entfalten und müsse sich jederzeit an ihrem Beitrag zur Lösung gesellschaftlicher Probleme messen lassen.

4 Die doppelte Politisierung des Diskurses ist augenfällig. Die Erwartung, daß „die Philologie“ (1.) sich von räumlichen, kulturellen und schon gar nationalen Bornierungen lösen solle, ist dringlich. Die Forderung sodann (2.), daß Philologie sich gesellschaftlich ausweisen müsse, ist unüberhörbar.

5 In Punkt (1) rate ich - Sie konnten das schon der Eingangsanekdote entnehmen - zur Bescheidenheit. Im übrigen gebe ich zu bedenken, daß man erst vergleichen kann, was man verstanden hat. Wenn aber der Eindruck nicht täuscht, fangen wir nur erst gerade an zu verstehen, was der Philologe tut, wenn er Philologe ist.

6 Auch Punkt (2) scheint mir auf einem Mißverständnis zu beruhen. Wenigstens sehe ich nicht, worauf ein philologischer „Netzwerker“ seinen Erkenntnis-Optimismus gründen könnte, solange seine besondere Arbeitsweise unverstanden bleibt.

7 Die Frage ist daher, glaube ich, wie grundsätzlich wir das Problem fassen wollen. Verstehen wir „Philologie“ nur eben als einen Sammelbegriff, in dem die unterschiedlichen Tätigkeiten (oder Praxen) des Philologen sich bündeln (lassen), also das Jagen und Sammeln, das Sichten und Ordnen, das Emendieren und Kommentieren, das Lesen und Schreiben? Oder glauben wir, daß Philologie noch etwas anderes ist, das sich in jedem einzelnen dieser Arbeitsschritte in einer noch $\mathrm{zu}$ bestimmenden und $\mathrm{zu}$ beschreibenden Weise zur Geltung bringt ${ }^{4}$ ? Mit anderen Worten: Ist Philologie die Funktion einer Praxis der Ermöglichung, Herstellung und Verteidigung von Sinn, oder ist sie sehr viel stärker in diesen Prozeß der Sinnverfertigung involviert, als es auf den ersten Blick scheinen mag5? Und wäre das Politische dann nicht etwas, das nicht erst in der Wendung der philologischen Tätigkeit nach außen in Erscheinung träte, sondern vielmehr immer schon dagewesen wäre - etwa im Vollzug der Moderation philologischer und literarischer Erkenntnis?

8 Wir sehen, wie "richtige“, intersubjektiv nachvollziehbare Positionen wie die von Christoph König und Julika Griem in ein falsches Licht rücken und vielleicht auch in Teilen „unrichtig“ werden, wenn sie verkürzt dargestellt werden - verkürzt um die Rechenschaftslegung oder Selbstaufklärung des philologischen Verfahrens ${ }^{6}$. König rekurriert häufiger auf Autoritätsmomente (die Autorität der Tradition, der Autoren 
und Gelehrten) und die Evidenz und Schlüssigkeit des ganz bestimmten Sinns der großen Werke. Griem verliert sich in den Formularen eines institutionellen Systemgedankens und bleibt die überzeugende Beschreibung des philologischen Kerngeschehens schon im Ansatz schuldig.

Es ist an der Zeit, auf einen zweiten großen ,homme Lillois' hinzuweisen, unseren Freund und Kollegen Alain Deremetz, dem die deutsche Debatte, von der ich nun ungebeten in einiger Ausführlichkeit berichtet habe, womöglich gar nicht einleuchten wird. Hat er in seinen großen Untersuchungen zur Autoreflexivität der klassischen und nachklassischen lateinischen Literatur die gesellschaftliche Frage nicht immer, oft $a$ fortiori, mitbedacht ${ }^{7}$ ? Deremetz verbindet Literaturwissenschaft und Soziologie auf so außergewöhnlich elegante Weise, daß ich mich schon oft gefragt habe, warum nicht gerade ein solches seltenes Beispiel für die tief- und weitsichtige Verknüpfung des Ästhetischen und des Politischen noch stärker Schule gemacht hat. Nun, es ist wahrscheinlich einfach zu intelligent. Und da wir uns gewöhnt haben, die Werke selbst befreundeter Wissenschaftler vorzugsweise konsumptiv, d.h. im Hinblick auf die Abschöpfung des für uns selbst Relevanten, zu lesen, ist es nicht weiter verwunderlich, daß so manches gelungene philologische ouvre kaum je in dem ihm eigenen Glanz erstrahlen kann.

Nun hat auch das gedrängte Lob den strategischen Nachteil, daß man den laudatus in eine Zone rückt, in die er vielleicht gar nicht hineingehört, in einen Raum „hors de critique“ gewissermaßen. Das wäre fatal. Mit Deremetz' Einsichten will gearbeitet sein. Man muß an sie anknüpfen, auf ihnen aufbauen dürfen. Und man muß fragen, wie sie sich zu anderen möglichen Positionen verhalten. Damit komme ich auf das Problem des Eingangs zurück, auf die Philologie, genauer auf die Unterscheidung einer vermeintlich idiosynkratischen, unpolitischen und einer vermeintlich offenen, diskurstüchtigen (interdisziplinären), politischen Philologie.

11 Ich deutete es schon an, daß ich diese Opposition solange für unplausibel und unproduktiv halte, wie nicht die Vertreter beider Standpunkte zu validen Aussagen darüber kommen, welches das Verhältnis der Sinnstiftungspotentiale der Philologie und der Sinnstiftungspotentiale der Texte ist. Sie bemerken zugleich, daß ich die Frage dieses Kolloquiums nach der angemessenen Methode auf jenes Feld verschiebe, das mir der Ort zu sein scheint, an dem - um in der technischen Sprache zu bleiben - die „Röhren“ der Texte und ihrer Erklärer „kommunizieren“, ich meine das Feld der Philologie.

Während Methoden, Zugänge oder approaches darauf ausgehen, die Texte auf eine bestimmte Weise und in einer bestimmten Richtung zu befragen, ohne sich in der Regel selbst problematisch zu werden, besteht die Philologie darauf, ihr eigenes Handeln in allem, was sie tut oder nicht tut, zu reflektieren. Sie untersucht dieses ihr Handeln oder Nichthandeln immer auch im Verhältnis zum Gegenstand der Untersuchung. Sie weiß, daß, was sie im Text erblickt hat, nicht unabhängig von diesem ihrem Sehen zu denken ist. Und sie gibt sich keinen Illusionen darüber hin, daß ihr eigenes Erkennen nicht ohne den Anstoß zu denken wäre, den sie im Umgang mit dem Text erfahren hat. Es ist dieses unvermeidliche Nahverhältnis, das die Philologie in den Augen ihrer Verächter verdächtig macht ${ }^{8}$. Philologie ist eine Politik der Intimität, die nichts mit Abschottung und Esoterik zu tun hat, sondern dem Umstand Rechnung trägt, daß die Produktion von Sinn nur dann zu haben ist, wenn der Strahl der Zeichen die deutende Netzhaut berührt. Radikal ist diese Philologie, weil sie dem Problem der Kontiguität nicht 
ausweicht, sondern, im Gegenteil, ihr Lesen schon beim ersten frontalen Zusammenstoß beginnen läßt ${ }^{9}$. Statt die in diesen An- und Abstoßungsszenen freigesetzte Energie zu neutralisieren - etwa in der Geste: „wir lassen den Text erst einmal auf uns wirken“ - nutzt sie die neuen Kräfte zur umfassenderen und gründlicheren Beschreibung des Vorgangs. Mit anderen Worten, sie erkennt schon im ersten, und vielleicht sollte man sagen: gerade im ersten Zusammenstoß das dynamische Potential der Zeichen, bevor diese sich in der sedierenden Synthesis zu Sinneinheiten geformt haben ${ }^{10}$. Für athematische Lektüren dieser Art habe ich in den letzten Jahren einige Beispiele geliefert ${ }^{11}$. Nota bene: Es geht nicht um die bildlichromantische Reformulierung phänomenologischer Binsenweisheiten, sondern darum, den Ort der Interpretation präziser zu bestimmen, als dies in der hierarchischen Sicht konventioneller Wissenschaftstheorie zuweilen geschehen ist. Nach dieser trägt der Interpret etwas an den Text heran oder zieht etwas aus ihm heraus, das sich als Sinn bestimmen läßt. Erkenntnistheoretisch gewendet, könnte man von einem doppelten Wiedererkennen sprechen, sei es, daß wir uns die Sache so denken, daß wir im Text ein von uns zuvor schon Erkanntes erkennen (eine Perspektive, die wenig Lust auf fleißige Anwendung macht), sei es, daß wir, gut Böckhisch, das im oder vom Text Erkannte noch einmal erkennen ${ }^{12}$. Beide Ansichten des Problems greifen zu kurz, wenn wir uns nur vergegenwärtigen, wie hier wie dort die Fiktion einer einsinnigen Richtung (Unidirektionalität) obwaltet. Böckh würde sich heute dem Vorwurf ausgesetzt sehen, er sei einem naiven Essentialismus aufgesessen, da er ja glaube, die Aufgabe der Interpretation sei es, das im Text Erkannte noch einmal zu erkennen. Aber auch der naseweise Konstruktivismus, der das Problem dadurch zu lösen sucht, daß er in allem Interpretieren nur Zuschreibungen erkennen will, führt hier nicht weiter ${ }^{13}$. Es ist doch gar nicht zu bestreiten, daß diese Zuschreibungen, wo sie nicht einer „idiotischen“ Phantasie entspringen, Häufigkeiten, Routinen, Praxen entwickeln, die - ungeachtet des Einflusses, den andere, vor allem natürlich frühere Zuschreibungen auf den Interpretationsprozeß nehmen mögen - eine gewisse Stringenz entwickeln. Das Kriterium für die Beurteilung dessen, was stringent ist, kann seriöserweise nicht die Setzung einer wie immer gearteten, jedenfalls in aller Regel außerhalb des Textes zu denkenden Autorenintention sein. Mit letzterer mag sich der Historiker oder Psychologe befassen. Nein, komplizieren wir das Geschäft nicht, indem wir zu den unübersehbar zahlreichen Ungewißheiten der Textkonstitution noch die Abgründe eines auktorialen Willens hinzunehmen ${ }^{14}$. Ein Kriterienkatalog zur Bearbeitung literarischer Texte müßte anders beschaffen sein.

Die lateinische Literatur ist für einen traditionellen Interpreten, genau genommen, ein hoffnungsloser Fall. Hier kann er weniger als in den meisten anderen Literaturen hoffen, zu bündigen Beschreibungen dessen zu kommen, was in den Texten geschieht. Das Problem verschärft sich in der augusteischen Literatur, in der aus der Sprachnot, die in Cicero und Lukrez noch ein prominentes Thema war, konsequent eine Stärke entwickelt wird. Die mangelnde Bestimmtheit der Aussagerichtung, die dem fehlenden Artikel und dem sparsamen Gebrauch von Hauptsatzkonjunktionen, vor allem aber dem weitgehend freien Satzbau geschuldet ist, wird nun bei gleichzeitiger Prägnanz der Nominal- und Verbalmorphologie in einen Vorteil verwandelt. Das Einzelwort erhält eine Aufmerksamkeit, die es in Konversationssprachen mit geschmeidigerem Satzduktus niemals haben kann. Vergil und Horaz und zuweilen auch Ovid und die Elegiker entwickeln eine beachtliche Virtuosität in der Moderation des Verhältnisses von nüchterner Denotation des Einzelworts und subversiver Umrüstung desselben zu 
einem $\mathrm{zu}$ vielfältigen Assoziationen einladenden Klangkörper. In einem solchen Arrangement kommt lange vor der Bestimmung eines Themas der Benennung ganz elementarer Relationen und Ordnungssignale große Bedeutung $\mathrm{zu}^{15}$. Um nur ein Beispiel herauszugreifen: Die Ideenwelt der augusteischen Elegie läßt sich aus dem Studium ihres Präpositionengebrauchs exakter entwickeln als aus unserer Kenntnis der Sozialgeschichte des zeitgenössischen Roms. Das sogenannte servitium amoris ist philologisch zunächst nur als grammatikalisches Arrangement zu haben. Aus der Untersuchung des Gegenstandsgebrauchs in Elegie 1, 3 lernen wir mehr über die Eigentümlichkeit des Tons dieser Gattung, als durch die wiederholte Darstellung des Figurenspiels zu gewinnen wäre ${ }^{16}$. Daß es im Eröffnungsgedicht der Oden-Sammlung des Horaz um das Problem einer Elevation geht, die ihre Rückbindung an die Phänomene der umgebenden Welt zu keinem Zeitpunkt preisgibt, erkennen wir erst bei der genauen Betrachtung der sprachlich-rhetorischen Bildschöpfungsverfahren ${ }^{17}$. Die Beispiele sind Legion. Das athematische Lesen scheint mir deshalb in hohem Maße zur Untersuchung der augusteischen Literatur geeignet, weil es gerade den gegen ein schnelles Verständnis sich sperrenden Elementen der Sprache eine neue Aufmerksamkeit zuteil werden läßt. Da es nicht zum schnellen Erfolg, zur Synthese kommen muß, kann es länger bei den Voraussetzungen der Entstehung einer Ordnung verweilen, die Semantik allererst möglich macht. ${ }^{18}$

Überhaupt scheint die augusteische Literatur Lesarten zu begünstigen, die sich nicht in der Vogelperspektive einrichten, sondern nah an den Fundamenten der Sprache operieren. Wenn die prinzipale These unseres Freundes, Alain Deremetz, richtig ist, hat die römische Literatur in kurzer Zeit einen Fundus an Ausdrucksformen hervorgebracht, die in besonderer Weise geeignet sind, die Selbstbegründungsprozeduren erst der römischen Republik, dann des Prinzipats „au miroir des muses “ $\mathrm{zu}$ spiegeln ${ }^{19}$. Diese Ausdrucks- oder besser: Darstellungsformen, die ich mit dem im Deutschen sehr geläufigen Begriff der „Denkfiguren“ belegen möchte, lese ich als die gewissermaßen nächsthöhere Stufe der Organisation literarischer Rede $^{20}$. Auf ihr haben sich die elementaren Bewegungsrichtungen (wie sie etwa in Präpositionen und Tropen angezeigt werden) in Einstellungen oder Perspektiven verwandelt. So sind sie fähig, jene von Deremetz geschilderte komplexe Poetik der Reflexivität in Gang zu bringen.

Nehmen wir das Beispiel der katábasis. Der Abstieg oder Rückgang zu den Quellen des Wissens und der Inspiration ist seit der Nekyia der Odyssee ein zentraler Baustein epischer, später auch dramatischer, lyrischer, philosophischer und satirischer Rede ${ }^{21}$. Deremetz hat ihm einen der schönsten Abschnitte seines großen Buches gewidmet ${ }^{22}$. Ich habe mich oft gefragt, worin die außerordentliche Anziehungskraft, worin der überragende Erfolg dieses alten Modells der reliefartigen, mitunter stufenleiterhaften Vertiefung gründen mag. Da ist natürlich zum einen die Möglichkeit, Tradition und Zusammenhang gerade dort zu generieren, wo der Bruch offensichtlich ist (die Welt der Lebenden, das Reich der Toten). Da ist aber zum anderen die Möglichkeit, das absolut Andere der Tradition zu gewinnen dadurch, daß die Gegenwart sich total aufs Spiel setzt. Sie geht ein in die Vergangenheit um den Preis, sich zu verlieren. Wenn sie zurückkehrt, kehrt sie stärker zurück. Aber da ist noch etwas anderes, das die Katabase zu einem Erfolgsmodell ohne gleiches macht.

16 Als ich vor Jahren daran ging, die Geschichte der Philologie aus Anekdoten, autobiographischen und memorialen Gesten großer Vertreter der Zunft zu deuten, 
stach die Prominenz katabatischer Topoi ins Auge ${ }^{23}$. Ulrich von WilamowitzMoellendorff scheute sich nicht, seine Methode der Philologie einem Gang in die Unterwelt $\mathrm{zu}$ vergleichen ${ }^{24}$. Hugh Lloyd-Jones hat daraus das Motto seiner

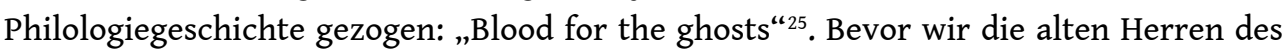
Mystizismus oder gar der Mystagogie zeihen, sollten wir uns darüber klar werden, daß hier in Bildern auch eine Erfahrung des Lesens ausgesprochen ist.

Verdeutlichen wir zum Abschluß das bisher Gesagte an einem Beispiel. Vor über zehn Jahren durfte ich schon einmal etwas $\mathrm{zu}$ einem Liller Kolloquium unserer Forschergruppe beitragen. Auch in dem „Blinde Mimesis“ überschriebenen Aufsatz ging es um die Unterweltsfahrt in Horaz c. 2, $13^{26}$. Ich habe Ihnen damals nicht die ganze Geschichte erzählt. Die Geschichte der „blinden Mimesis“ klingt ja doch zunächst nach einer Verlustgeschichte: Kunde statt Autopsie, Imagination statt Erinnerung, Poesie statt Geschichte. Man kann sie aber auch als die Geschichte einer philologischen Erfahrung lesen: Wir beobachten, wie das lyrische Ich seinen jähen Tod durch den Sturz des morschen Holzes auf seinem Gütchen imaginiert. Wir sehen es „hinabgefahren in das Reich des Todes“, wo es in der verstörenden Begegnung mit den Gestalten der Unterwelt und vor allem mit seinen berühmten frühgriechischen Vorläufern Sappho und Alkaios seine poetische Zukunft gewinnt. Aber all dies sagt der Dichter ja nicht. Er zeigt uns nur die fragmentierten Bilder, die wir aus einem Erfahrungsraum, den das Gedicht nur zitiert, ergänzen ${ }^{27}$. Carmen 2, 13 präsentiert uns einen ungedeckten Wechsel. Und doch ergreift es uns, weil das Verfahren, das uns vor die Augen rückt, unser eigenes philologisches Verfahren ist. Es beschreibt den unbeschreiblichen Moment, wo sicheres Wissen in Unsicherheit umschlägt. Die Hadesfahrt - und das meine ich nicht allegorisch - ist des Philologen Alltag ${ }^{28}$. Unser Lesen ist ein Tauchgang, bei dem die Bewohner der Tiefe, die unsren Besuch nicht erbeten haben, auf allen Seiten an uns vorüberfliegen. Die einen erscheinen dicht vor uns im hellen Licht der Lampe, andere verschwinden, kaum daß wir sie gewahr wurden, und stieben an uns vorbei - zurück in die von keinem Licht erhellte, schwarze Nacht. Jedes Lesen ist ein anderes Lesen. Nie sind wir sicher, daß die Expedition gelingt. Und wenn wir gelesen haben, wenn wir mithin an die Oberfläche zurückgekehrt sind, stehen wir vor dem fast noch größeren Wagnis, von dem zu sprechen, was wir gesehen haben. Haben wir etwas gesehen? Quam paene ... vidimus, sagt das lyrische Ich am schwärzesten Punkt seiner Reise (v. 21). Des Dichters „Ich“ hat nichts gesehen. Die „Erfahrung“, die es mitteilt, ist blinde Mimesis, die Schaffung eines Szenarios, das auf keiner Erfahrung gründet ${ }^{29}$. Das unterscheidet es von uns Philologen. Wir steigen wirklich hinab. Wenn wir uns konzentrieren, müssen wir so viele Außeneinflüsse wie möglich abwehren. Wir blenden Umwelten aus, um die Konturen des Textes zu sehen und die Hand an die pulsierenden Ströme zu legen, die dem Text diese Kontur verschaffen. Wir spüren, wenn wir konzentriert sind, die kleinsten Risse und Fugen und suchen diese Spuren im Gedächtnis zu bewahren. Wir bestimmen selbst, wann wir im Nachlassen der Konzentration zu lesen aufhören, um an die Textoberfläche zurückzukehren. Jetzt stellt sich die Frage: Haben wir etwas gesehen und, wenn ja, was? Welchen Status hat das Gesehene? Wer wollte es überprüfen? Kann man es überprüfen? Oder ist nicht die einzige Form der Überprüfung die Wiederholung des Tauchgangs? Nicht nur gibt es keine Gewähr, daß wir Glauben finden, es ist auch schon fast unmöglich, daß wir selbst das Gesehene so darstellen können, daß wir den Text, zu dem wir unsere Leseeindrücke verdichten, in allen Punkten getreu nach dem Vorbild des Geschauten gestalten können ${ }^{30}$. 

unseres Eindrucks. Sind wir mit der schweren Ausrüstung unserer Kenntnis der Literaturgeschichte hinabgestiegen oder nur mit dem Leichtgepäck uneingeschränkten Zutrauens $\mathrm{zu}$ - unserer Intuition?

athematische Lesen ist kein wiedererkennendes Lesen. Auch der Horazische Unterweltsfahrer erkennt ja nichts wieder. In der Imagination der Unterweltsszene bildet er entschlossen seine Zukunft. Am Singen der lesbischen Sänger gewinnt er auch das Kriterium seiner eigenen Dichtung. Das Urteil über die Kunst der Sappho und des Alkaios wird ja nicht von Richtern (wie dem Unterweltsweisen Aeacus) gesprochen. Das Staunen der umstehenden Schatten entscheidet. Beider Gesänge sind - so die bewundernde Menge - „heiligen Schweigens wert“ (sacro digna silentio, v. 29). „Mehr“ aber „trinkt mit $\mathrm{Ohr}(\mathrm{en})$ “ (bibit aure, v. 32) das Schulter an Schulter sich drängende Volk die Lieder von Schlachten und Tod der Tyrannen. Die Philologie der Vielen ist unmittelbar und sinnlich. Sie ist nicht banal. In der Erfahrung der Kunst verkehren sich auch älteste Gewißheiten ${ }^{31}$. Die hundertköpfige Bestie läßt die Ohren herabhängen. Die Nattern im Haar der Rachegöttinnen kommen zur Ruhe. Prometheus und des Pelops Erzeuger lassen sich vom schmeichelnden Klang der Lieder über ihre Fron hinwegtäuschen. Orion denkt nicht länger daran, Löwen und Luchse zu scheuchen. Das lyrische Ich liest die infernale Veranstaltung mit der Besessenheit des Archäologen, der in den steinernen Urkunden seine eigenen Wurzeln erschließen zu können hofft.

Während die Imagination des lyrischen Ichs in der Unterwelt verbleibt, muß die Interpretation am Ende „auf den Boden der Tatsachen“ zurückkehren. Nein, sie darf sich zu keiner Zeit von ihm entfernen. Dies gelingt ihr umso leichter, wenn sie sich nicht in den Deutungsangeboten des Schattenreiches verliert, sondern nur ihrem eigenen Kompaß vertraut. Immun gegen die sirenische Versuchung des Nah- und Nächstliegenden erhält sie sich - wie die Gefährten des Odysseus mit ihren wachsverschlossenen Ohren - ihre eigene Gangart und sucht auf den Grund der Rede zu kommen. Im entschlossenen Abstieg berührt sie sich mit der Dekonstruktion. Die Katabase ist ihr freilich nicht Ziel der (Irr)Fahrt, sondern - gut odysseisch - Mittel zum Zweck ${ }^{32}$. Sie kehrt „auf den Boden der Tatsachen“ zurück und liest und erkennt in den Bruchstücken der Unterweltsfahrt den Traumtext des „wirklichen“ Textes: die Philologie.

Was soll das heißen: „den Traumtext des, wirklichen Textes, die Philologie“?! Ich bin Ihnen, glaube ich, eine Erklärung schuldig. „Philologisch“ nenne ich nicht nur die Arbeit am Text, sondern auch das, was im Text arbeitet und macht, daß es von uns bemerkt und erkannt werden kann. Ohne die Philologie des Textes, d.h. ohne den differenzierten Mechanismus der zur Erzeugung des Textes ineinander greifenden Sprachspiele gäbe es nichts, dem unsere Philologie begegnen könnte. Das ist weder Wortklauberei noch Metaphysik. Erkennen wir endlich die Membran, auf die wir stoßen, wenn wir philologisch lesen! Das lyrische Ich in c. 2, 13 des Horaz tut, z.B., nichts anderes. Es führt uns in bald großen, bald knappen, fast jähen Gesten sein Deuten vor. Erst deutet es die üblen Machinationen seines Beinahe-Verhängnisses, dann deutet es, was ihm erspart geblieben. Die doppelte Konjektur über die Ursache des Bösen wie über das knapp vermiedene Erlebnis aber führt auf einen Wahrheitskern, der im Gedicht selbst nur wie in einem Spiegel erscheint. Jetzt verstehen wir auch, warum die Stimme des Ichs am Ende im Orkus verbleibt. Sie hat ihre Bestimmung gefunden. Die Philologie des Philologen aber hat das Problem der Philologie des Textes geerbt. 
Wahrheit kann sie - erkenntnistheoretisch gesprochen - nur anamnetisch oder eben als konjekturale Philologie ermitteln, ästhetisch gewendet kann sie Eigentliches nur uneigentlich oder wie durch einen Spiegel sagen. Muß ich noch hinzufügen, daß solche Erkenntnis und solche Ästhetik nur im Augenblick zu haben ist ${ }^{33}$ ? Horaz carmen kreist um den Augenblick, in dem sich die Dinge zur schönen Einsicht wenden. Im Augenblick auch entsteht die philologische Erkenntnis. Sie trägt die Schlacken der Kontingenz. Auch das macht sie zum methodischen Abenteuer.

Mein Plädoyer geht auf eine Philologie von unten, eine Philologie, die sich nicht scheut, in die Abgründe der literarischen Rede zu tauchen, diese schwer übersehbaren Zonen, die sich der unkomplizierten Herstellung von Sinn verschließen. Ich plädiere für Tauchfahrten ohne vorherige Zielbestimmung. Die Begegnung mit dem Anderen des Textes, dem Bodensatz der Zeichen vor allem, die zur Ordnung drängen, muß vorbehaltlos sein. Auch wo/wenn wir nicht fündig werden, wird die fundlose Fahrt doch nicht vergeblich sein, weil sie uns den Respekt vor der Schwierigkeit der Aufgabe lehrt.

Wir haben die Hadesfahrt des lyrischen Ichs mit dem Abenteuer des philologischen retour sur soi-même verglichen und den Augenblick des Umschlags von sicherem Wissen in Nichtwissen als den heikelsten Punkt dieser Reise benannt. An diesem Wendepunkt, so sagten wir, springt die nichtgemachte Erfahrung in die Rekonstruktion einer Geschichte um, die die Basis für eine dichterische Zukunft resp. das philologische Urteil werden soll. Politisch ist die deszendentale Philologie, weil sie in der Dichtung den Punkt der absoluten Freiheit benennt (es ist in einigen der schwierigsten Oden des Horaz zugleich das Zentrum der Konstruktion) und bei seiner Bergung auch die Gefahr des Scheiterns auf sich nimmt. In Horaz c. 2, 13 ist das Politische dann doch auch thematisch geworden: Der Augenblick des Tod und Leben verschmelzenden quam paene ... vidimus (den wir als Moment höchster Freiheit beschrieben haben) setzt noch eine andere, politische Freiheitserfahrung frei: „Trinken die Schulter an Schulter sich drängenden Schatten“ nicht besonders Alkaios " politische Lieder ${ }^{34}$ „mit Ohr(en)“? Und ist es nicht diese politische Kunst, die macht, daß sich am Ende die inbegrifflichen Gewißheiten der Untererde in sich selbst verkehren? Oder, mit anderen Worten: Wann hätte Politik auf Erden mehr erreicht, als in dieser Unterweltsfahrt von der politischen Kunst des Alkaios berichtet wird? Prometheus und der erzbetrügerische Vater des Pelops, Tantalos, lassen sich dulci ... sono (v. 38) über ihre Fron hinwegtäuschen. Qual verwandelt sich in Genuß. Mit einem Male sehen wir wieder „das richtige im falschen Leben“. Die augusteische Literatur ist reich an Texten, deren politische Brisanz nicht offen zutage liegt. Der primäre Impuls des Politischen erschließt sich in der athematischen Lektüre mitunter leichter, als wenn man das Verständnis des Textes erst bei den Themen und Motiven beginnen läßt. Ich glaube übrigens auch (aber das wäre schon Stoff für einen zweiten Vortrag), daß es die nicht- oder vorthematischen Bewegungen der Sprache und Texte sind, die ihnen diese unheimliche Gegenwart bis in unsere Tage sichern. Die Bausteine der Rede veralten eben nicht - und schon gar nicht die aus ihnen geronnenen, von Alain Deremetz so glänzend beschriebenen Denkfiguren. Sie sind keinen Konjunkturen ausgesetzt. Die augusteische Literatur aber ist nach meiner bescheidenen Einschätzung die alphabetische Kultur, die das Prinzip der radikalen, bodennahen, ihre Themen bis auf den Grund zurücklesenden Konstruktion am stärksten verinnerlicht hat. Nur so konnte sie nach meiner Überzeugung diese beispiellose Erfolgsgeschichte schreiben.* 


\section{BIBLIOGRAPHIE}

ALT, P.: Die Verheißungen der Philologie, Göttingen 2007.

Arweiler, A.: Souveränität und Einschließung. Catull, Cicero und Vergil über Macht, die Expansion der Herrschaft und die Autorität der Literatur, in: Ders./B. Gauly (Hrsg.): Machtfragen. Zur kulturellen Repräsentation und Konstruktion von Macht in Antike, Mittelalter und Neuzeit, Stuttgart 2008, S. 19-78.

Arweiler, A./Möller, M. (Hrsg.): Vom Selbst-Verständnis in Antike und Neuzeit. Notions of the Self in Antiquity and Beyond, Berlin 2008 (= Transformationen der Antike 8).

Arweiler, A.: Römische Literaturen und die Grenzen der Philologie, in: S. Winko/ F. Jannidis/G. Lauer (Hrsg.): Grenzen der Literatur: zu Begriff und Phänomen des Literarischen, Berlin/New York 2009, S. 545-83.

Bohnenkamp, A./Bremer, K./Wirth, U./Wirtz, I. (Hrsg.): Konjektur und Krux. Zur Methodenpolitik der Philologie, Göttingen 2010.

Blumenberg, H.: Wirklichkeitsbegriff und Wirkungspotenzial des Mythos, in: M. Fuhrmann (Hrsg.): Terror und Spiel. Probleme der Mythenrezeption, München 1971, S. 11-66 (= Poetik und Hermeneutik 4).

Böckh, A.: Encyklopädie und Methodologie der philologischen Wissenschaften, hrsg. v. E. Bratuschek, 2. Auflage, besorgt von R. Klussmann, Leipzig ${ }^{2} 1886$ (1877).

Bohrer, K.H.: Plötzlichkeit. Zum Augenblick des ästhetischen Scheins, Frankfurt a.M. ${ }^{3} 1998$ (1981). Deremetz, A.: D'Homère à Virgile, ou le retour aux trois fonctions, in: RHR 204 (1987), S. 115-28.

-: La prière en représentation à Rome. De Mauss à la pragmatique contemporaine, in: RHR 211 (1994), S. 141-65.

----------: Le Miroir des muses: Poétiques de la réflexivité à Rome, Villeneuve d'Ascq (Nord) 1995.

-: La terre inspirée et le poète-paysan, in: Noesis 4 (2000), S. 155-79.

: Énée aède. Tradition auctoriale et (re)fondation d'un genre, in: E.A. Schmidt (Hrsg.): L'histoire littéraire immanente dans la poésie latine, Vandœuvres-Genève 2001, S. 143-82 (= Entretiens XLVII).

-: La Sibylle dans la tradition épique à Rome : Virgile, Ovide et Silius Italicus, in: M. Bouquet/F. Morzadec (Hrsg.): La Sibylle. Parole et représentation, Rennes 2004a.

: Tradition, vraisemblance et autorité fictionnelle, in: Dictynna 1 (2004b), S. 5-26.

-: L'histoire du genre épique dans les catabases de Virgile, d'Ovide et de Silius Italicus, in: J.P. Schwindt (Hrsg.): La représentation du temps dans la poésie augustéenne - Zur Poetik der Zeit in augusteischer Dichtung. Internationales Kolloquium der Forschergruppe „La poésie augustéenne“, Heidelberg 2005, S. 111-22.

Les récits d'Achéménide et de Macarée dans le livre XIV des Métamorphoses d'Ovide, in: VL 183/184 (2011), S. 150-60.

Emmrich, T.: Hier freut sich der Tod, dem Leben zu helfen. Katabatische Ordnungen der Literatur, in: C.D. Haß/ E.M. Noller (Hrsg.) 2015, S. 173-211. 
Griem, J.: Wir Philologen - heute ohne Aura, in: FAZ, 7.10.2015.

Gumbrecht, H.U.: Die Macht der Philologie. Über einen verborgenen Impuls im wissenschaftlichen Umgang mit Texten, Frankfurt a.M. 2003.

-: Diesseits der Hermeneutik. Über die Produktion von Präsenz, Frankfurt a.M. 2004.

: Präsenz, hrsg. u. mit einem Nachwort v. J. Klein, Berlin 2012.

Hamacher, W.: Entferntes Verstehen. Studien zu Philosophie und Literatur von Kant bis Celan, Frankfurt a.M. 1998.

----------: Für - die Philologie, in: J.P. Schwindt (Hrsg.) 2009b, S. 21-60.

-: 95 Thesen zur Philologie, Holderbank 2010.

Haß, C.D./Noller, E.M. (Hrsg.): Was bedeutet Ordnung - was ordnet Bedeutung? Zu

bedeutungskonstituierenden Ordnungsleistungen in Geschriebenem, Berlin/Boston 2015

(= Materiale Textkulturen 10. Schriftenreihe des Sonderforschungsbereichs 933).

Hummel, P.: Mœurs érudites. Étude sur la micrologie littéraire (Allemagne, XVI-XVIIIe siècles), Genf 2002 (= Histoire des idées et critique littéraire 395).

-: Philologus auctor. Le philologue et son œuvre, Bern u.a. 2003 (= Sapheneia. Beiträge zur Klassischen Philologie 8).

(Hrsg.): Metaphilology. Histories and languages of philology, Paris 2009.

Kelemen, P./Kulcsár Szabó, E./Tamás, A. (Hrsg.): Kulturtechnik Philologie. Zur Theorie des Umgangs mit Texten, Heidelberg 2011 (= Bibliothek der klassischen Altertumswissenschaften 131).

Klingner, F.: Ohnmacht und Macht des musischen Menschen, in: Ders.: Studien zur griechischen und römischen Literatur, Zürich/Stuttgart 1964, S. 325-33.

König, C. (Hrsg.): Das Potential europäischer Philologien. Geschichte, Leistung, Funktion, Göttingen 2009 (= Philologien. Theorie - Praxis - Geschichte 1).

-: Achtung vor den fremden Werken. Zuerst lesen wir hingerissen, dann lesen wir analytisch. Über die philologische Aufgabe, den Sinn schwieriger Texte zu verstehen, in: FAZ, 23.09.2015.

Lepper, M.: Philologie zur Einführung, Hamburg 2012.

Lloyd-Jones, H.: Blood for the Ghosts: Classical Influences in the Nineteenth and Twentieth Centuries, Baltimore 1982.

Möller, M.: Rhetorik und Philologie. Fußnoten zu einer Theorie der Aufmerksamkeit, in: J.P. Schwindt (Hrsg.) 2009b, S. 137-159.

Most, G. (Hrsg.): Aporemata, 6 Bd., Göttingen 1997-2002.

Paulus, J.: Theoretische Philologie. Annäherung an eine disziplinäre und methodische Leerstelle, in: C.-F. Berghahn/R. Stauf (Hrsg.): Philologie als Kultur. Die Germanisch-Romanische Monatsschrift 1909-2009, Heidelberg 2009, S. 33-50.

Platthaus, I.: Höllenfahrten. Die epische katábasis und die Unterwelten der Moderne, München 2004.

Schäfer, E.: Horaz nach Actium, in: WJA 13 (1987), S. 195-207. 
Schlaffer, H.: Poesie und Wissen. Die Entstehung des ästhetischen Bewußtseins und der philologischen Erkenntnis, Frankfurt a.M. 1990.

Schwindt, J.P.: Blinde Mimesis. Über Ordo und Kontingenz in der lateinischen Traditionsbildung (Horaz und Petron), in: Dictynna 1 (2004a), S. 157-74.

-: Dislocatio temporis. Struktur und Ereignis in Horaz' Lyrik, in: W. Lange/ Ders./K. Westerwelle (Hrsg.): Temporalität und Form. Konfigurationen ästhetischen und historischen Bewußtseins, Heidelberg 2004b, S. 77-93.

-: Schwarzer Humanismus. Brauchen wir eine neue Alte Philologie?, in: Merkur 60 (2006), S. 1136-50.

-: Vom Phantasma zur Denkfigur. Das Neue bei den Griechen und Römern, in: Merkur 62 (2008), S. 793-803.

: Thaumatographia, or „What is a Theme?", in: P. Hardie (Hrsg.): Paradox and the Marvellous in Augustan Literature and Culture, Oxford 2009a, S. 145-62.

(Hrsg.): Was ist eine philologische Frage? Beiträge zur Erkundung einer theoretischen Einstellung, Frankfurt a.M. 2009b.

-: Traumtext und Hypokrise. Die Philologie des Odysseus, in: Ders. (Hrsg.): Was ist eine philologische Frage? Beiträge zur Erkundung einer theoretischen Einstellung, Frankfurt a.M. 2009c, S. 61-81.

-: „Unkritik“ oder das Ideal der Krise. Vom Ende und vom Anfang philologischer Kritik, in: P. Kelemen/E. Kulcsár Szabó/Á. Tamás (Hrsg.): Kulturtechnik Philologie. Zur Theorie des Umgangs mit Texten, Heidelberg 2011a, S. 239-48.

: Paradise lost. Warum die Klassische Philologie ihre Standards und Normen überdenken muß, in: Journal of Literary Theory 5 (2011b), S. 245-49.

: Philologie des Lebens 1911 Philologie des Todes, in: Ders. (Hrsg.): Edmund Hoppe. Mathematik und Astronomie im Klassischen Altertum, Bd. 1, mit einem Nachwort von M. Asper, Heidelberg 22013 (2011c), S. 5-60.

-: Über Genauigkeit, in: Ders. (Hrsg.): Edmund Hoppe. Mathematik und Astronomie im Klassischen Altertum, Bd. 2, mit einer fachlichen Einführung von M. Asper, Heidelberg 2012, S. 269-301.

--------: Ordo and insanity. On the pathogenesis of Horace's Ars poetica, in: MD 72 (2014), S. 197-216.

: (Radikal)Philologie, in: T. Meier/M.R. Ott/R. Sauer (Hrsg.): Materiale Textkulturen. Konzepte - Materialien - Praktiken, Berlin/München/Boston 2015, S. 235-43.

-: Thaumatographia oder Zur Kritik der philologischen Vernunft. Vorspiel: Die Jagd des Aktaion (Ovid, Metamorphosen 3, 131-259), Heidelberg 2016 (= Bibliothek der klassischen Altertumswissenschaften 150).

-: Der Karneval des Properz. Zur Objektepistemologie der augusteischen Literatur, in: K.P. Hofmann/M. Hilgert/H. Simon (Hrsg.): Objektepistemologien. Zur Vermessung eines transdisziplinären Forschungsraums, Berlin 2017a.

: Das athematische Lesen, erscheint voraussichtlich 2017b in: L. Banki/M. Scheffel (Hrsg.): Lektüren. Positionen zeitgenössischer Philologie. 
Über das philologische Erkennen - Sobre o conhecimento filológico, in: I.T. Cardoso/ J.P. Schwindt (Hrsg.): Wörter für eine Theorie der Philologie - Palavras para uma teoria da filologia, Heidelberg 2017c.

Steinfeld, T.: Der leidenschaftliche Buchhalter. Philologie als Lebensform, München/Wien 2004.

Wilamowitz-Moellendorf, U.v.: Greek Historical Writing and Apollo. Two lectures delivered before the University of Oxford June 3 and 4, 1908, translated by G. Murray, Oxford 1908 (Nachdr.: Chicago 1979).

\section{NOTES}

1. Eröffnungsvortrag des 12. Kolloquiums der Internationalen Forschergruppe „La poésie augustéenne“. Der Doppelcharakter des Textes als hommage auf einen großen Gelehrten und als Erkundungsfahrt (oder Tauchgang) zu einer seiner Leitideen wurde mit Bedacht nicht verändert.

2. Siehe König 2015: „Die Philologie kennt einen ihr eigenen Königsweg: Sie sucht die Antwort auf die Frage nach einer eigenen Wissenschaftstheorie in der Reflexivität ihrer Gegenstände. Weil die Poesie denkt, kann ihr der Interpret gedanklich auf den Spuren sein. Der Interpret trachtet danach, der poetischen Reflexivität gerecht zu werden“.

3. Siehe Griem 2015.

4. Vgl. die anregenden Untersuchungen etwa von Schlaffer 1990, Most 1997-2002, Hummel 2002, 2003 u. 2009, Gumbrecht 2003, Steinfeld 2004, Alt 2007, König 2009, Hamacher 2010, Bohnenkamp/Bremer/Wirth/Wirtz 2010, Kelemen/Kulcsár Szabó/Tamás 2011 und Lepper 2012.

5. Die methodologisch heikelste Frage ist die nach der Involvenz des philologischen Fragens in den Prozeß der Erkenntnisverfertigung der Literatur. Ihr ist meine Untersuchung Thaumatographia oder Zur Kritik der philologischen Vernunft (2016) gewidmet.

6. Zur Notwendigkeit einer Theorie der Philologie, die sich Rechenschaft von den methodologischen Voraussetzungen ihrer Arbeit ablegen kann, vgl. Paulus 2009, Schwindt 2009b, 2009c, 2011 b u. 2012, Bohnenkamp/Bremer/Wirth/Wirtz 2010 u. Kelemen/Kulcsár Szabó/Tamás 2011.

7. So denkt Deremetz schon in der Einleitung zu „Le miroir des muses“ die Begriffe „poésie“ und ” societé “ zusammen (vgl. Deremetz 1995, S. 11-17). Zu einer „[...] littérature qui réfléchit sur les conditions de possibilité de sa propre création [...]“ (S. 23) bemerkt er: „si cette littérature exprime elle-même explicitement ou allégoriquement 'le fait qu'elle est le résultat d'une construction', alors elle pourra être dite réflexive“ (S. 24). Eine beispielhafte Lesart für diese theoretische Annahme findet sich bei Deremetz 2005. Siehe auch Deremetz 1994.

8. Schon der Name der Philologie verweist auf eine Disposition, die v.a. von Hamacher 2009 nach dem Vorgang Schlegels und Nietzsches - als Baustein für eine Theorie der Philologie in Anspruch genommen wird: Philologie als philía, „als eine Neigung zur Sprache [...], als eine Zuwendung und eine Näherung [...]" (S. 29). Mit dieser Nähe geht nach Hamacher jedoch immer auch Entfernung und Distanzierung einher: „Je näher die Philologie ihrer Sache rückt, desto ferner tritt sie zurück“ (S. 29; vgl. schon die Aufsatzsammlung „Entferntes Verstehen“: Hamacher 1998). Es ist damit diese „Doppelbewegung“, die die Philologie charakterisiert. Vgl. auch Schwindt 2009c, S. $62 \mathrm{f}$.

9. Zur Begründung der „Radikalphilologie“ siehe Schwindt 2006 und 2015.

10. Im Unterschied zu einem unmittelbar sinnstiftenden Lesen, wie es hermeneutische oder rezeptionsästhetische Modelle nahelegen, wird ein radikalphilologischer Leser versuchen, die erste Berührung mit dem Text zu beobachten und zu beschreiben. Auch Gumbrecht empfiehlt mit seinem „Präsenz“-Modell einen Modus der Beschreibung des ästhetischen Erlebnisses als „Oszillieren (und mitunter auch als Interferenz) zwischen »Präsenzeffekten« und 
"Sinneffekten«" (Gumbrecht 2004, S. 18). Vor der Interpretation und damit - in Gumbrechts Worten - „diesseits der Hermeneutik“ findet so Kommunikation zwischen den Gegenständen des Textes und ihrem Betrachter statt, deren Beobachtung Aufgabe des (von uns geforderten) athematischen Lesens sein muß (vgl. auch Gumbrecht 2012). Hamacher 2009 fasst die Gefahr, die vom „Phantasma des Semantizismus, es gebe originäre und suisuffiziente Bedeutungen“, ausgeht, im prägnanten Bild des „Tod[es] des Wortes in der Bedeutung“ (S. 26). In Schwindt 2011a wird die Aussetzung und Aufschiebung des Urteils als genuin philologischer Akt beschrieben. In der „Unkritik“ finden „Literatur und Philologie soweit zusammen, daß die eine Institution die andere immer schon vorausdenkend antizipiert, ohne daß dadurch die jederzeit mögliche Entscheidung verunmöglicht wird“. Indem Philologie so zunächst von der bestimmenden Kritik zurücktritt, wird ein sinn-offenes Beschreiben und Beobachten möglich. Vgl. auch Möller 2009, die der Philologie die spezifische Möglichkeit einräumt, „sich gegenüber Sinn und Bedeutung neutral" verhalten zu können (S. 159).

11. Siehe z.B. Schwindt 2004a, 2004b, 2014 u. $2017 b$.

12. Siehe Böckh 1886, S. $10 \mathrm{f}$.

13. Es ist vielmehr notwendig, die Erkenntnis des Philologen mit der Erkenntnis des Textes zusammenzudenken. In meinem demnächst erscheinenden Beitrag „Über das philologische Erkennen - Sobre o conhecimento filológico“ (Schwindt 2017c) wird die Erkenntnisleistung der Philologie so als „Katalysator im Prozeß der Freilegung [...] der Selbsterkenntnis des Textes“ beschrieben. Vgl. auch meine Kritik des Konstruktivismus in Schwindt 2011d, S. 272f. Zur besonderen Erkenntnisleistung der Philologie vgl. auch Schwindt 2009a, 2009b. Siehe auch Schwindt 2015, S. 243: „Der Philologie eignet vielmehr eine Form des Erkennens, die die Erkenntnis der Texte zur Entfaltung bringt und dann am wirksamsten ist, wenn sie bis zur Unkenntlichkeit im Text und seiner Nachbildung aufzugehen scheint".

14. Wo diese als literarisch-technische Strategien der Produktion oder Simulation eines AutorenIchs an der Textoberfläche sichtbar werden, müssen wir ihnen selbstverständlich unsere Aufmerksamkeit widmen. Siehe hierzu v.a. die Untersuchungen von Arweiler/Möller 2008 u. Arweiler 2008 u. 2009.

15. Über die prägnante athematische Disposition der Texte besonders der augusteischen Literatur siehe das Kapitel „Wer schützt die athematische Literatur?“ in Schwindt 2012, S. 296-98.

16. Vgl. meine Untersuchung dieser Properzischen Elegie in Schwindt 2017a.

17. Zum „Denkbild der Erhebung“ in Horaz c. 1, 1 siehe Schwindt 2015 (siehe S. 239).

18. Siehe auch Haß/Noller 2015.

19. Siehe z.B. Deremetz 1995, S. 145, u. vgl. Deremetz 1987, 1994, 2000, 2001, 2004 b u. 2011.

20. Zu „Denkfiguren“ bzw. „Denkbildern“ in der lateinischen Dichtung siehe Schwindt 2008 u. 2015.

21. Emmrich 2015 beschreibt die katabáseis der antiken Literatur als Strategien der Sicherung von Wissen, wenn Ordnung und Orientierung in Gefahr geraten sind: „Dass dem limen ultimum ein Quantum Wissen und infolgedessen auch ein Stück Leben abgerungen werden kann, ist keine originäre Erkenntnis der Pathologie [...]. Diese heuristische Erkenntnis gehört bereits in der Antike zum impliziten Wissen literarischer Hadesfahrten. In der Odyssee und der Aeneis wird die räumlichexistenzielle Letztgrenze von Lebenden durchbrochen, die damit die Ordnung stören, sich allerdings gerade dadurch in den Dienst derselben stellen“ (S. 177). Zu Potenzial und Sonderstatus der homerischen Nekyia siehe Platthaus 2004, S. 94ff.

22. Siehe das Kapitel „La catabase d'Enée: Enéide 6“ in Deremetz 1995, S. 156-171, u. vgl. Deremetz 2004a u. 2005.

23. Siehe Schwindt 2011c, u. vgl. Schwindt 2009c, S. 63f., u. Schwindt 2016, S. $18 f$.

24. Siehe Wilamowitz-Moellendorf 1908, S. 25: „The tradition yields us only ruins. The more closely we test and examine them, the more clearly we see how ruinous they are; and out of ruins no whole can be built. The tradition is dead; our task is to revivify life that has passed away. We 
know that ghosts cannot speak until they have drunk blood; and the spirits which we evoke demand the blood of our hearts. We give it to them gladly; but if they then abide our question, something from us has entered into them [...]".

25. Lloyd-Jones 1982.

26. Schwindt 2004a.

27. Nach Klingner 1964 genügen „einige Namen [...], um das Bild des Totenreiches gegenwärtig zu machen“" (S. 330).

28. Zur katabatischen Dimension der Philologie („Philologie als Hadesfahrt“) vgl. Hamacher 2010 (Thesen 71 u. 74), Schwindt 2011c u. 2014 sowie Emmrich 2015. Platthaus 2004 beschreibt die Unterweltsmetaphorik als Teil des Narrativs der Selbstbeschreibung der sich im 18. und 19. Jahrhundert konstituierenden "Wissenschaften der Tiefe" (Archäologie, Geologie, Psychoanalyse), S. 11f.

29. Siehe Schwindt 2004a, S. 174.

30. Das sind Unwägbarkeiten, die der alte Böckh übersah. Er rechnete nicht mit der Unzulänglichkeit des Menschen, seiner Unfähigkeit, für das, was er sieht, adaequate Formen des Ausdrucks zu finden. Noch weniger aber rechnete er mit den Grenzen der Darstellbarkeit der Dinge in der Sprache.

31. Schon Klingner 1964 benennt die „Umkehrung“ als zentrales Motiv der Horazischen Ode, geht dabei aber von thematologischen Beobachtungen und Annahmen aus: „Denn was geschieht denn in dem Gedicht, wenn man es als Vorgang, als Bewegung auffaßt, wie es doch offenbar verstanden sein will? Etwas verwandelt sich in sein Gegenteil [...]“ (S. 332). In dieser Lesart folgt ihm unter anderen Schäfer 1987. In meinem Text zur „Blinden Mimesis“ habe ich versucht zu zeigen, wie in einer radikalen, athematischen Lesart die „Verkehrung ins Gegenteil“ als konstitutiv „für die Gedichtstruktur im ganzen“ gelesen werden kann (vgl. Schwindt 2004a, S. 172f.). So kann, was Klingner und Schäfer als zentrales Thema des Gedichtes erkennen, schon vorher, athematisch, als strukturelles Zentrum beschrieben werden.

32. Hans Blumenberg 1971 spricht treffend vom „poetische(n) Obligatorium der Unterweltsfahrt des epischen Helden“ (S. 21).

33. Zur theoretischen Grundlegung der Darstellung und Beschreibung des Plötzlichen als "Ausdruck und Zeichen von Diskontinuität und Nichtidentischem“, das als strukturelles Zentrum des Textes das Ästhetische vom Nichtästhetischen scheidet, siehe Bohrer 1998, S. 7. Zur chronopoetischen Konstruktion des Augenblicks in c. 2, 13 vgl. Schwindt $2004 \mathrm{~b}$.

34. [...] sed magis / pugnas et exactos tyrannos / densum umeris bibit aure volgus (v. 30-32). Siehe auch Schäfer 1987, der die Präsenz des Politischen in c. 2, 13 in den Kontext einer imitatio des Alkaios durch Horaz einordnet. Dieser fühle sich seinem Vorbild „vor allem in seinen politischen Oden verbunden" (S. 201, vgl. S. 195). Indem Schäfer hier direkt an konkrete politische Ereignisse erinnert und das Politische somit thematisch zu fassen versucht (ebd.), tut er freilich den zweiten vor dem ersten Schritt, der darin bestünde, der Frage nachzugehen, wie das Politische vor jeder motivischen Semantisierung gleichsam im „Untergrund“ des Textes präsent ist.

*. Ich danke meinen studentischen Mitarbeiterinnen Chiara Cavazzani, Johanna Victoria Kaiser und Miriam Schilling. Es ist ein wahres Glück, mit ihnen zu arbeiten! Es ist ihr Verdienst, daß im Anmerkungsapparat manches konsolidiert und vertieft werden konnte, was im Fließtext meiner Lille-Rede nur flüchtig berührt war. 


\section{RÉSUMÉS}

Der Beitrag versucht die Frage nach den Prinzipien und Methoden einer zeitgemäßen Interpretation augusteischer Dichtung so grundsätzlich wie möglich zu fassen. Die Philologie wird als ein Feld von Einstellungen und Handlungsoptionen beschrieben, die noch keine Entscheidung für eine bestimmte Methode der Interpretation nahelegen. Politisch ist die Philologie nicht, weil sie Lesarten generiert, die sich entsprechend semantisieren lassen, sondern weil sie im fortlaufenden Prozeß der Rechenschaftslegung und Selbstaufklärung die prinzipielle Unverfügbarkeit des Textes sicherstellt. Die Rechenschaftslegung nun geschieht nicht vor oder nach, sondern in der Auseinandersetzung mit den Texten. Das "athematische Lesen“ feit die Philologie gegen die unkritische Begegnung mit dem Eigenen im Fremden.

Es war Alain Deremetz, der in seinen Untersuchungen zur katabatischen Form auf die Bedeutung der Unterweltsfahrten im Prozeß der Selbstverständigung der römischen Kultur verwiesen hat. Die „Philologie von unten“ ist geeignet, im Verfahren der Rekonstruktion der literarischen Rede die Fragmente jener radikalen Politik zu bergen, die am Grunde der Texte liegt. Neues Licht fällt auch auf „die Form des Erscheinens der Literatur“, i.e. die Philologie: In Horaz c. 2, 13 ist sie der „Traumtext des ,wirklichen“ Textes“. Sie ist die Netzhaut, auf der sich das philologische Erkennen in der Erkenntnis des literarischen Textes bricht. In der Denkfigur der katábasis erschließt sich dem Dichter wie dem Philologen das proprium ihres Schreibens: daß sie nämlich Erkenntnis nur um den Preis der Bereitschaft zur Selbstaufgabe gewinnen und „Eigentliches nur uneigentlich oder wie durch einen Spiegel sagen“ können. Der heikelste Punkt der doppelten Hadesfahrt ist der „Augenblick des Umschlags von sicherem Wissen in Nichtwissen“. Wenn eine nichtgemachte Erfahrung zum Zentrum der Konstruktion des Gedichts werden kann, ist zugleich der (paradoxe) Punkt benannt, in dem die radikale Politik und die radikale Philologie konvergieren: Es ist die uneinholbare Erfahrung der absoluten Freiheit.

\section{INDEX}

Schlüsselwörter : Horaz c. 2. 13, Alain Deremetz: Literaturwissenschaft und Soziologie, das Politische der Philologie, das Politische der Literatur, die augusteische Dichtung als „athematische Literatur“, das „athematische Lesen“, die katábasis als Denkfigur, Literatur und Erkenntnis, Radikale des Politischen und radikale Philologie, Erfahrung und Nichterfahrung, die „absolute Freiheit“, die „Philologie von unten“

\section{AUTEUR}

\section{JÜRGEN PAUL SCHWINDT}

Heidelberg 